\title{
A Cross-Sectional Online Survey of HIV Pre-Exposure Prophylaxis Adoption Among Primary Care Physicians
}

\author{
Oni J. Blackstock, MD, MHS' , Brent A. Moore, $P h D^{2}$, Gail V. Berkenblit, MD, $P h D^{3}$, \\ Sarah K. Calabrese, $P h D^{4}$, Chinazo O. Cunningham, $M D, M S^{7}$, David A. Fiellin, $M D^{2,4}$, \\ Viraj V. Patel, MD, MPH' ${ }^{7}$ Karran A. Phillips, MD ${ }^{5}$, Jeanette M. Tetrault, MD², Minesh Shah, MD 6 , and \\ E. Jennifer Edelman, $M D, M H S^{2,4}$
}

\begin{abstract}
${ }^{1}$ Montefiore Medical Center/Albert Einstein College of Medicine, Bronx, NY, USA; ${ }^{2}$ Yale University School of Medicine, New Haven, CT, USA; ${ }^{3}$ Johns Hopkins University, Baltimore, MD, USA; ${ }^{4}$ Yale University School of Public Health, New Haven, CT, USA; ${ }^{5} \mathrm{NIDA}$-Intramural Research Program, NIH, Baltimore, MD, USA; UUniversity of Illinois-Chicago, Chicago, IL, USA.
\end{abstract}

BACKGROUND: Among health care providers, prescription of HIV pre-exposure prophylaxis (PrEP) has been low. Little is known specifically about primary care physicians (PCPs) with regard to PrEP awareness and adoption (i.e., prescription or referral), and factors associated with adoption.

OBJECTIVE: To assess PrEP awareness, PrEP adoption, and factors associated with adoption among PCPs.

DESIGN: Cross-sectional online survey conducted in April and May 2015.

RESPONDENTS: Members of a national professional organization for academic primary care physicians $(n=$ 266).

MAIN MEASURES: PrEP awareness, PrEP adoption (ever prescribed or referred a patient for PrEP [yes/no]), provider and practice characteristics, and self-rated knowledge, attitudes, and beliefs associated with adoption.

KEY RESULTS: The survey response rate was $8.6 \%$ (266/2093). Ninety-three percent of respondents reported prior awareness of PrEP. Of these, 34.9 \% reported PrEP adoption. In multivariable analysis of provider and practice characteristics, compared with non-adopters, adopters were more likely to provide care to more than 50 HIV-positive patients (vs. 0, aOR = 6.82, $95 \%$ CI 2.0622.52). Compared with non-adopters, adopters were also more likely to report excellent, very good, or good selfrated PrEP knowledge (15.1 \%, $33.7 \%$, $30.2 \%$ vs. $2.5 \%$, $18.1 \%, 23.8 \%$, respectively; $p<0.001$ ) and to perceive PrEP as extremely safe (35.1\% vs. $10.7 \% ; p=0.002)$. Compared with non-adopters, adopters were less likely to perceive PrEP as being moderately likely to increase risk behaviors ("risk compensation") (12.8 \% vs. $28.8 \%$, $p=0.02$ ).

CONCLUSIONS: While most respondents were aware of PrEP, only one-third of PrEP-aware PCPs reported adoption. Adopters were more likely to have experience providing HIV care and to perceive PrEP as extremely safe, and were less likely to perceive PrEP use as leading to risk

Electronic supplementary material The online version of this article (doi:10.1007/s11606-016-3903-z) contains supplementary material, which is available to authorized users.

Received April 18, 2016

Revised August 29, 2016

Accepted October 5, 2016

Published online October 24, 2016 compensation. To enhance PCP adoption of PrEP, educational efforts targeting PCPs without HIV care experience should be considered, as well as training those with HIV care experience to be PrEP "clinical champions". Concerns about safety and risk compensation must also be addressed.

KEY WORDS: HIV prevention; pre-exposure prophylaxis; primary care physicians; implementation.

$\mathrm{J}$ Gen Intern Med 32(1):62-70

DOI: $10.1007 / \mathrm{s} 11606-016-3903-z$

(c) Society of General Internal Medicine 2016

\section{INTRODUCTION}

Although new diagnoses of human immunodeficiency virus (HIV) in the United States have declined over the past decade, 40,000 individuals continue to be diagnosed with HIV each year. ${ }^{1}$ Pre-exposure prophylaxis (PrEP) represents an innovative HIV prevention strategy that has the potential to further reduce new HIV infections. ${ }^{2}$ PrEP involves HIV-negative individuals taking antiretroviral medications and attending routine visits with a health care provider. ${ }^{2}$ To date, multiple studies have demonstrated the efficacy of PrEP across a range of risk groups. ${ }^{3-7}$ While there have been concerns raised, particularly regarding renal and bone-induced adverse effects, the effects have been largely reversible. ${ }^{7,8}$ Furthermore, data regarding the potential for HIV resistance has been reassuring. ${ }^{7}$ In 2014, the Centers for Disease Control and Prevention (CDC) released guidelines regarding PrEP (tenofovir disoproxil fumarate/emtricitabine [Truvada $\left.{ }^{\circledR}\right]$ ) for persons at substantial risk for HIV infection. ${ }^{2}$ The CDC estimates that 1.2 million individuals are eligible for PrEP based on sexual and drug use behaviors, yet only about 80,000 unique individuals have been prescribed PrEP since its 2012 approval by the Food and Drug Administration. ${ }^{9,10}$ Moreover, studies suggest that only a minority of U.S. health care providers report having ever prescribed PrEP. ${ }^{11-17}$

Health care providers play a central role in the implementation of new health-related technologies, given their access to individuals or populations that might benefit most. ${ }^{18}$ Primary 
care physicians (PCPs), in particular, are optimally positioned to provide PrEP, given their focus on health promotion, disease prevention, and longitudinal care. Additionally, PCPs primarily deliver care to HIV-negative individuals, some of whom may be at substantial HIV risk, and therefore PrEPeligible. ${ }^{17,19}$ Despite the potentially important role of PCPs in implementing PrEP (i.e., incorporating PrEP into clinical practice), earlier studies of providers' PrEP-related attitudes and potential role in PrEP adoption (i.e., commitment to and initial use of PrEP, defined in this study as a prescription of or referral for PrEP) ${ }^{20}$ have focused mainly on HIV specialists, ${ }^{12,15,21-24}$ such as infectious disease (ID) physicians, and/or have been limited to specific geographic regions of the U.S. ${ }^{13,16,25-29}$ No published studies of PrEP adoption have focused exclusively on general internists, which is notable, given that they are abundant, geographically distributed throughout the U.S., and uniquely positioned to offer PrEP to persons at high risk of HIV infection.

Therefore, we sought to 1) characterize the level of PrEP awareness and adoption (i.e., PrEP prescription or referral) among PCPs, and 2) examine provider and clinical practice characteristics as well as provider self-rated PrEP-related knowledge, attitudes, and beliefs associated with PrEP adoption.

\section{METHODS}

\section{Respondents and Procedures}

From April through May 2015, we conducted an anonymous online survey of members of the Society of General Internal Medicine (SGIM), a national professional organization for academic general internists. We recruited potential participants using a listserv for SGIM members (with reminder emails sent weekly for 6 weeks) as well as direct e-mail messaging. At the time of the survey, SGIM had 3093 active members who were attending physicians, fellows, or residents. All members are automatically registered to the SGIM listserv. Recruitment emails included a brief description of the study, with a hyperlink to the online survey. As we aimed to survey PCPs who could potentially prescribe or refer patients for PrEP, we specifically recruited those involved in direct outpatient care. Therefore, eligible study respondents were SGIM members who currently provided direct clinical care or supervise trainees in the outpatient setting. Upon survey completion, we offered respondents entry into a raffle to win one of two iPads. The study was administered by Albert Einstein College of Medicine and Yale School of Medicine, and received institutional review board (IRB) exemption from both institutions.

Of 363 initiated surveys with completed eligibility questions, 64 respondents were excluded because they were not SGIM members, and 12 were excluded because they did not provide direct clinical or supervising trainees in an outpatient setting. Of the remaining 287 surveys, 21 had missing data for the primary outcome, leaving 266 eligible surveys.

\section{Data Collection}

We administered the PCP PrEP Survey to respondents using an online survey tool (Qualtrics $\left.{ }^{\circledR}\right)$. The PCP PrEP Survey was informed by a literature review of health care providers' PrEPrelated knowledge, attitudes, and behaviors, ${ }^{11-16,21-23,25-32}$ an existing survey instrument, ${ }^{33}$ and feedback from community members involved in HIV prevention research. It was developed and pilot-tested in an iterative fashion by the authors, most of whom are PCPs who provide HIV care and/or researchers who conduct HIV bio-behavioral research (Online Appendix). The 57-item survey, which was delivered in English, included provider sociodemographic, clinical, and practice characteristics, self-rated knowledge, attitudes, and beliefs about PrEP, and PrEP adoption.

\section{Measures}

Outcome of Interest. PrEP adoption, our primary outcome, was defined as ever having prescribed or referred a patient for PrEP (yes/no). PrEP adoption was based on participants' responses to the following two questions: "Have you ever prescribed PrEP for a patient?" (yes/no) or "Have you ever referred a patient for PrEP (e.g., to a PrEP provider or HIV clinic)?" (yes/no). We chose this composite measure, as some PCPs may practice in clinical settings where referral to a designated PrEP provider or HIV clinic (vs. prescription by the internists themselves) is considered standard of care for provision of PrEP.

Independent Variables. Provider characteristics collected included age (estimated by year of birth), race/ethnicity, gender identity, sexual orientation, current role (attending physician vs. trainee [fellow or resident]), and percentage of time allocated to specified activities (direct patient care, research, medical education, administration, and other activity).

Clinical practice characteristics collected included region of the country in which the participant's clinic was located (West, Midwest, South, Northeast), rurality/urbanicity of clinic location (urban, suburban, rural), type of clinical setting (clinic at an academic medical center, clinic at a public hospital, community health center, clinic at VA hospital, other), and estimated number of HIV-positive clinic patients for whom the participant provided care $(0,1-10,11-20,21-50,>50)$.

Self-rated knowledge of PrEP and its potential side effects were measured on a five-point Likert scale (poor $=1$, fair $=2$, good $=3$, very good $=4$, excellent $=5$ ). Attitudes and beliefs about PrEP's effectiveness, safety, and impact on risk behaviors, and likelihood of prescribing PrEP in the next 6 months were assessed by items with possible responses on a four-point Likert scale (not at all $=1$, slightly $=2$, moderately $=3$, extremely $=4$ ).

\section{Statistical Analysis}

First, to characterize the analytic sample, we used descriptive statistics. Next, to compare characteristics between PrEP adopters and non-adopters, we used chi- 
square tests and multivariate analysis of variance (MANOVA). To assess the association between provider/clinical practice characteristics and PrEP adoption, we performed logistic regression. Variables that were significant at $p<0.1$ in unadjusted analysis were included in a final multivariable logistic regression model. We considered all independent variables with $p<0.05$ in the final model as significant. The associations between provider/clinical practice characteristics and PrEP adoption are presented as odds ratios (OR), along with associated $95 \%$ confidence intervals (CI). In sensitivity analyses, we excluded respondents with zero HIV-positive patients and conducted additional analyses to examine the association of direct patient care time with PrEP adoption and knowledge, attitudes, and beliefs. Statistical analyses were performed using SPSS version 21 software (IBM Corp., Armonk, NY, USA).

\section{RESULTS}

The response rate was estimated to be $8.6 \%$ (266 completed surveys/3093 SGIM members invited to participate). Of 266 respondents, $246(92.5 \%)$ had previously heard of PrEP and thus were included in the analysis for PrEP adoption.

\section{Characteristics of Analytic Sample}

Among our analytic sample $(n=246)$, the mean age was 40.9 (SD 9.6) years (Table 1). Most were white (73\%), female (62\%), heterosexual (91\%), and attending physicians (79\%). On average, the sample spent $41 \%$ of their time involved in direct patient care. Clinics were located mainly in the Northeast $(50 \%)$, and in urban areas $(85 \%)$. Almost two-thirds (68\%) of respondents practiced at clinics at academic medical centers, and $75 \%$ provided outpatient care for at least one HIV-positive patient. Over one-third (34.9\%) had ever

Table 1 Provider and Practice Characteristics by PrEP Adoption

\begin{tabular}{|c|c|c|c|c|c|}
\hline & $\begin{array}{l}\text { Overall sample } \\
(n=246)\end{array}$ & $\begin{array}{l}\text { Adopters }(n= \\
\text { 86) }\end{array}$ & $\begin{array}{l}\text { Non-adopters } \\
(n=160)\end{array}$ & $\begin{array}{l}\text { Unadjusted odds ratio } \\
(95 \% \text { CI })\end{array}$ & $\begin{array}{l}\text { Adjusted odds ratio } \\
\text { (95\% CI) }\end{array}$ \\
\hline Age (years), mean (SD) & $40.9(9.6)$ & $39.7(9.4)$ & $41.6(9.7)$ & $0.98(0.95-1.01)$ & \\
\hline \multicolumn{6}{|l|}{ Race, $n(\%)$} \\
\hline White & $162(73)$ & $56(72)$ & $106(73)$ & Ref & \\
\hline Black & $13(6)$ & $3(4)$ & $10(7)$ & $0.57(0.15-2.15)$ & \\
\hline Asian/Asian American & 39 (17) & $15(19)$ & $24(17)$ & $1.18(0.58-2.44)$ & \\
\hline Other & $9(4)$ & $4(5)$ & $5(3)$ & $1.51(0.39-5.87)$ & \\
\hline Hispanic ethnicity, $n(\%)$ & $15(7)$ & $5(6)$ & $10(7)$ & $1.09(0.36-3.03)$ & \\
\hline \multicolumn{6}{|l|}{ Gender, $n(\%)$} \\
\hline \multirow{2}{*}{\multicolumn{6}{|c|}{ Sexual orientation, $n(\%)$}} \\
\hline & & & & & \\
\hline Heterosexual & $210(91)$ & $73(91)$ & $137(91)$ & $1.01(0.39-2.64)$ & \\
\hline \multirow{2}{*}{\multicolumn{6}{|c|}{ Role, $n(\%)$}} \\
\hline & & & & & \\
\hline Attending physician & $182(79)$ & $70(86)$ & $112(75)$ & $2.16(1.04-4.50)$ & $1.88(0.89-3.99)$ \\
\hline Trainee & $49(21)$ & $11(14)$ & $38(25)$ & Ref & Ref \\
\hline \multicolumn{6}{|c|}{ Percentage of time allocation, mean (SD) } \\
\hline Direct patient care & $41.2(29.2)$ & $43.2(26.9)$ & $40.1(30.3)$ & $0.45 *$ & \\
\hline Research & $22.0(29.7)$ & $18.5(27.4)$ & $23.8(30.8)$ & $0.19 *$ & \\
\hline Medical education & $21.4(19.3)$ & $22.0(19.2)$ & $21.0(19.3)$ & $0.70 *$ & \\
\hline Administration & $13.7(18.1)$ & $12.9(16.4)$ & $14.2(18.9)$ & $0.61 *$ & \\
\hline Other & $1.9(8.5)$ & $3.4(12.7)$ & $1.0(4.7)$ & $0.52 *$ & \\
\hline \multicolumn{6}{|l|}{ Region of country, $n(\%)$} \\
\hline West & $39(17)$ & $18(22)$ & $21(14)$ & Ref & \\
\hline Midwest & $30(13)$ & $10(12)$ & $20(13)$ & $0.63(0.25-1.61)$ & \\
\hline South & $47(20)$ & $12(15)$ & $35(23)$ & $0.43(0.18-1.01)$ & \\
\hline Northeast & $117(50)$ & $41(51)$ & $76(50)$ & $0.68(0.35-1.32)$ & \\
\hline \multicolumn{6}{|c|}{ Rurality/urbanicity of practice, $n(\%)$} \\
\hline Urban & $202(85)$ & $72(89)$ & $130(86)$ & Ref & \\
\hline Suburban & $25(12)$ & $8(10)$ & $17(11)$ & $0.85(0.35-2.07)$ & \\
\hline Rural & $6(2)$ & $1(1)$ & $5(3)$ & $0.36(0.04-3.15)$ & \\
\hline \multicolumn{6}{|c|}{ Type of clinical setting, $n(\%)$} \\
\hline & $158(68)$ & $56(69)$ & $102(67)$ & Ref & \\
\hline \multicolumn{6}{|l|}{ medical center } \\
\hline 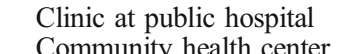 & $23(10)$ & $10(12)$ & $13(9)$ & $1.40(0.58-3.40)$ & \\
\hline $\begin{array}{l}\text { Community health center } \\
\text { Clinic at VA hospital }\end{array}$ & $18(9)$ & $7(9)$ & $15(10)$ & $0.85(0.33-2.21)$ & \\
\hline $\begin{array}{l}\text { Clinic at VA hospital } \\
\text { Other }\end{array}$ & $\begin{array}{l}14(6) \\
16(7)\end{array}$ & $\begin{array}{l}2(2) \\
6(7)\end{array}$ & $\begin{array}{l}12(8) \\
10(6)\end{array}$ & $\begin{array}{l}0.30(0.07-1.40) \\
1.09(0.38-3.17)\end{array}$ & \\
\hline \multicolumn{6}{|c|}{ Number of clinic patients with HIV, $n(\%)$} \\
\hline 0 & $59(25)$ & $15(18)$ & $44(29)$ & Ref & Ref \\
\hline $1-10$ & $123(53)$ & $41(51)$ & $82(54)$ & $1.47(0.73-2.94)$ & $1.43(0.71-2.88)$ \\
\hline $11-20$ & $18(8)$ & $7(9)$ & $11(7)$ & $1.87(0.61-5.69)$ & $1.83(0.60-5.62)$ \\
\hline $21-50$ & $14(6)$ & $5(6)$ & $9(6)$ & $1.63(0.47-5.63)$ & $1.65(0.46-5.87)$ \\
\hline$>50$ & $18(8)$ & $13(16)$ & $5(3)$ & $7.63(2.33-24.98)$ & $6.82(2.06-22.52)$ \\
\hline
\end{tabular}

$\%$ represents column percentage

$S D$ standard deviation, CI confidence interval

* $p$ values for the MANOVA, evaluating all time allocation variables together 
prescribed or referred a patient for PrEP. Specifically, $15.4 \%$ had only prescribed PrEP, $14.6 \%$ had only referred a patient for PrEP, and $4.9 \%$ had done both.

\section{Provider and Practice Characteristics Associated with PrEP Adoption}

In unadjusted analysis, being an attending physician (vs. trainee, $\mathrm{OR}=2.16,95 \% \mathrm{CI} 1.04-4.50$ ) and providing care to more than $50 \mathrm{HIV}$-positive patients (vs. $0, \mathrm{OR}=7.63,95 \% \mathrm{CI} 2.33$ 24.98) were associated with higher odds of PrEP adoption (Table 1). No other provider or clinical practice characteristics were associated with PrEP adoption. In the final multivariable model including only current role (attending vs. trainee) and number of HIV-positive clinic patients, only providing care to more than 50 HIV-positive patients (vs. 0, adjusted odds ratio $[\mathrm{aOR}]=6.82,95 \%$ CI 2.06-22.52) remained significantly associated with PrEP adoption. In sensitivity analyses, we found similar results (data not shown).

\section{Provider Self-Rated Knowledge, Attitudes, and Beliefs Associated with PrEP Adoption}

Compared with non-adopters, adopters were more likely to report excellent, very good, or good self-rated knowledge of $\operatorname{PrEP}(p<0.001$; Fig. 1$)$ and its side effects $(p<0.001$; Fig. 2$)$, and to report being extremely likely to prescribe PrEP in the next 6 months $(p<0.001$; Fig. 3$)$. Most adopters and nonadopters perceived PrEP as moderately safe; however, adopters were more likely than non-adopters to perceive PrEP as extremely safe ( $p=0.002$; Fig. 4$)$. A minority of adopters and non-adopters perceived PrEP as being moderately likely to increase risk behaviors; adopters were less likely than non-adopters to endorse this belief ( $p=0.02$; Fig. 5). Perceived effectiveness was not associated with adoption status ( $p=0.20$; Fig. 6).

\section{DISCUSSION}

We found that the vast majority of PCPs in our study sample were aware of PrEP. However, of these, only one-third had ever prescribed or referred a patient for PrEP, with about equal proportions reporting either. In multivariable analysis of provider and clinical practice characteristics, only providing care to more than $50 \mathrm{HIV-positive} \mathrm{clinic} \mathrm{patients} \mathrm{remained} \mathrm{associ-}$ ated with PrEP adoption. Compared with non-adopters, adopters were more likely to report greater self-rated knowledge of PrEP and its side effects, to perceive PrEP as extremely safe, and to report greater perceived likelihood of prescribing PrEP in the future. Compared with adopters, non-adopters were more likely to perceive PrEP as leading to an increase in risk behaviors ("risk compensation"). We did not find a difference in perceived effectiveness by adoption status.

Our study's findings regarding awareness and adoption of PrEP among PCPs represent an important contribution to the growing body of literature on the role of health care providers in PrEP implementation. While the CDC estimates that one-third of primary care providers are unaware of PrEP, ${ }^{17}$ the vast majority of PCPs in our study reported an awareness, a proportion similar to that in two other geographically focused U.S.-based studies that recruited ID/HIV specialists as well as primary care providers. ${ }^{16,28}$ The proportion of our study respondents reporting PrEP adoption (35\%) was among the highest of any published study. Most published studies on PrEP adoption were cross-sectional in design, recruited exclusively ID/HIV specialists or a combination of specialists and primary care providers via listservs, and utilized online surveys. ${ }^{11-17}$ These studies found that $4-32 \%$ of providers reported ever prescribing PrEP. In a recently published study of primary care clinicians, which included general

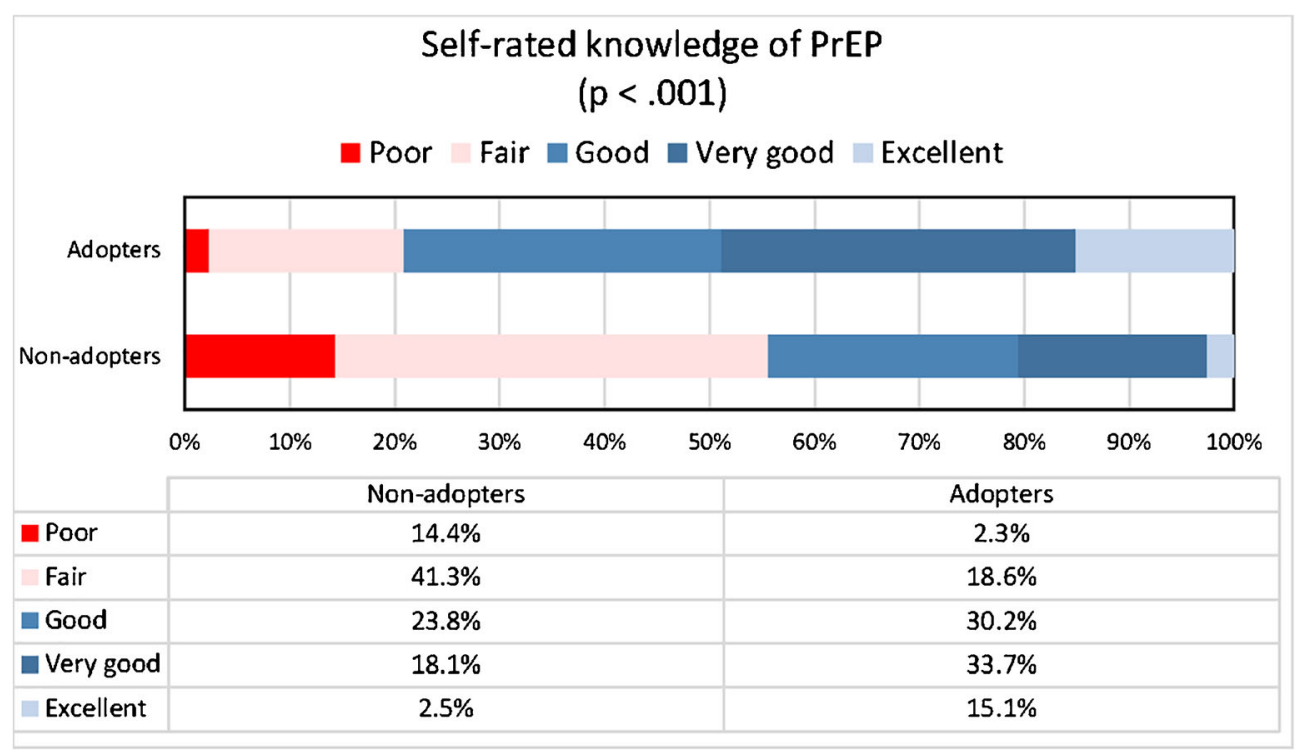

Figure 1 Self-rated knowledge of PrEP by adoption status. 


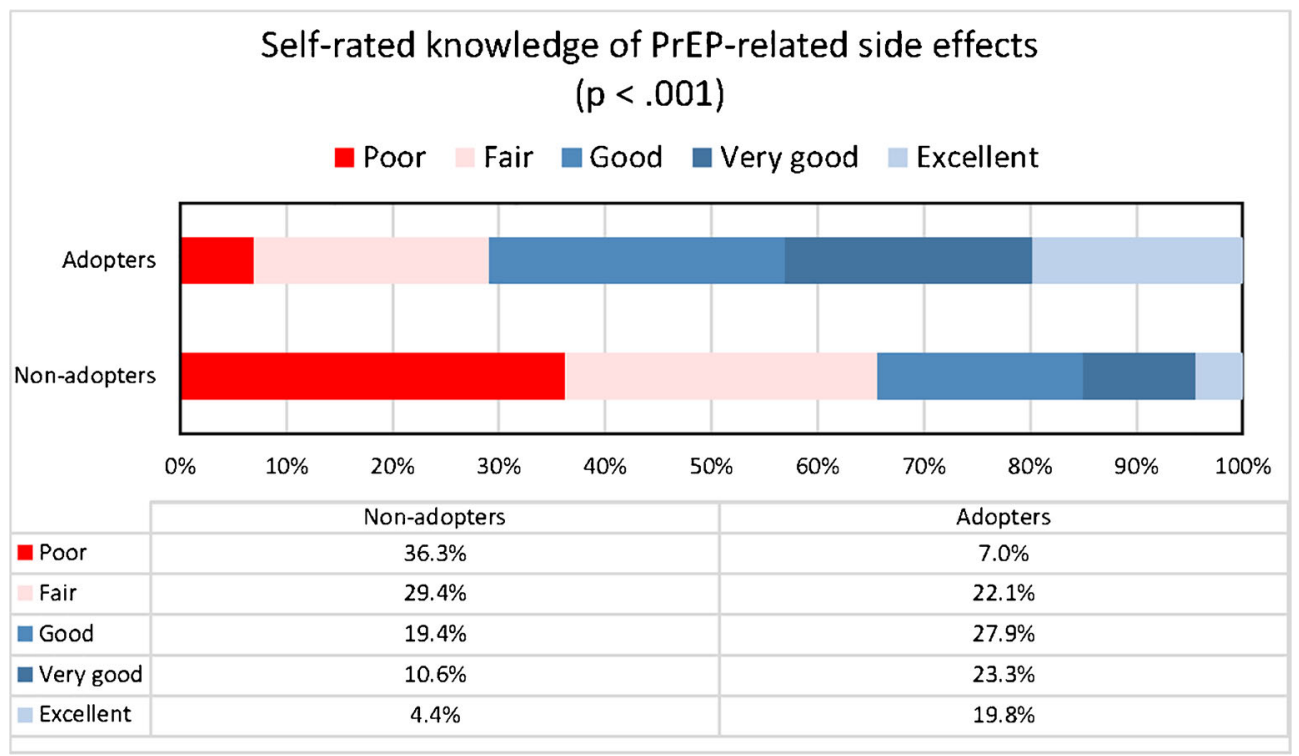

Figure 2 Self-rated knowledge of PrEP-related side effects by adoption status.

internists, family medicine physicians, obstetrician/ gynecologists, and nurse practitioners, $7 \%$ reported having prescribed PrEP. ${ }^{17}$ Although we used a composite measure of PrEP adoption (i.e., prescription or referral), as some primary care clinics may refer to an outside provider for PrEP-related services, even if we restricted our outcome to only those who ever prescribed PrEP, the proportion would be similar to or higher than all but one of the prior studies. Potential reasons for higher PrEP awareness and adoption in our study compared to others include response bias, sampling bias, and temporal trends including increasing attention to PrEP in the lay press and scientific journals, as well as outreach to providers by health departments nationwide.

Our findings suggest that providing care to more than 50 HIV-positive patients may be associated with a specific interest in HIV prevention and treatment. Due to the relatively high prevalence of co-existing substance use disorders among persons with HIV, it is possible that PCPs who provide HIV care to a relatively large number of HIV-positive patients may be more comfortable or familiar with harm reduction approaches such as PrEP. ${ }^{34,35}$ Results of studies have been mixed, with some showing a positive association between the number or proportion of HIV-positive patients for which a clinician provides care and surrogate measures of PrEP adoption, ${ }^{12,31}$ while others have shown no association. ${ }^{13,14,23}$

Our findings suggest that providers' concerns about the increase in risk behaviors and the safety of PrEP continue to be potential barriers to PrEP adoption. Fears that patient risk compensation (i.e., increase in sexual risk behavior due to a perceived reduction in HIV susceptibility) could offset the protective benefit of PrEP has been a prominent concern

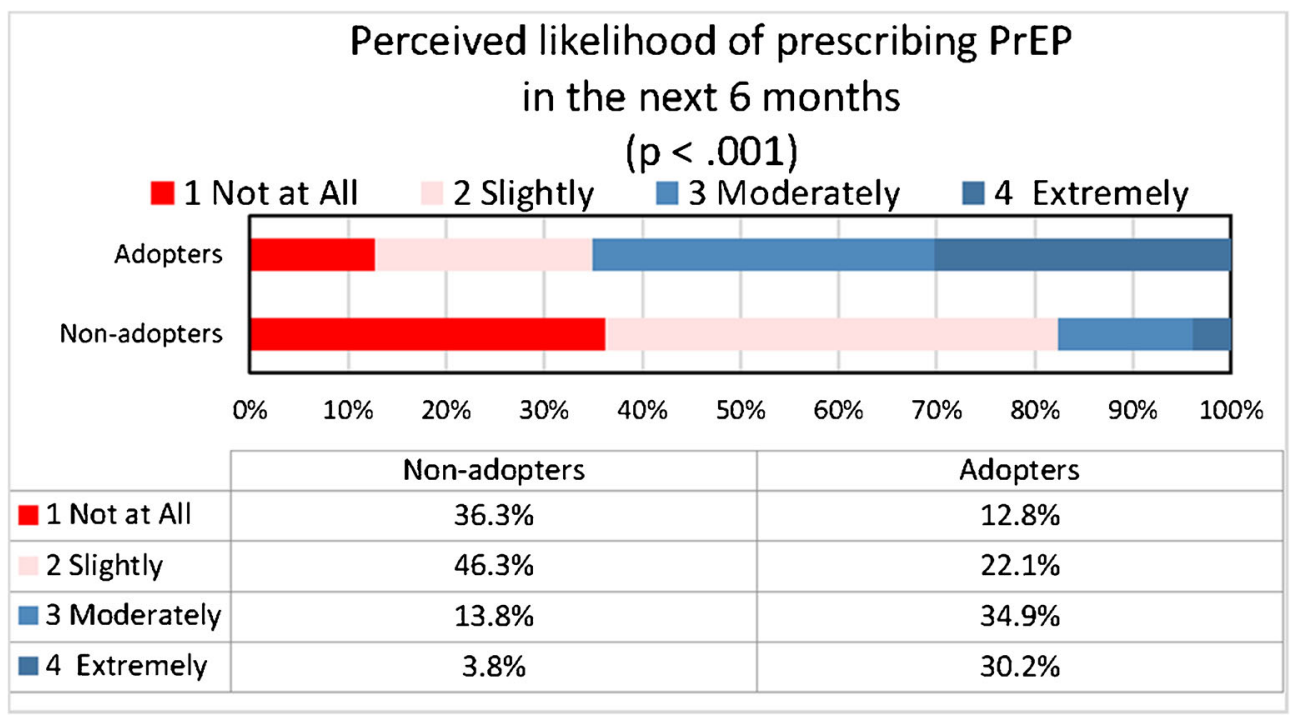

Figure 3 Perceived likelihood of prescribing PrEP by adoption status. 


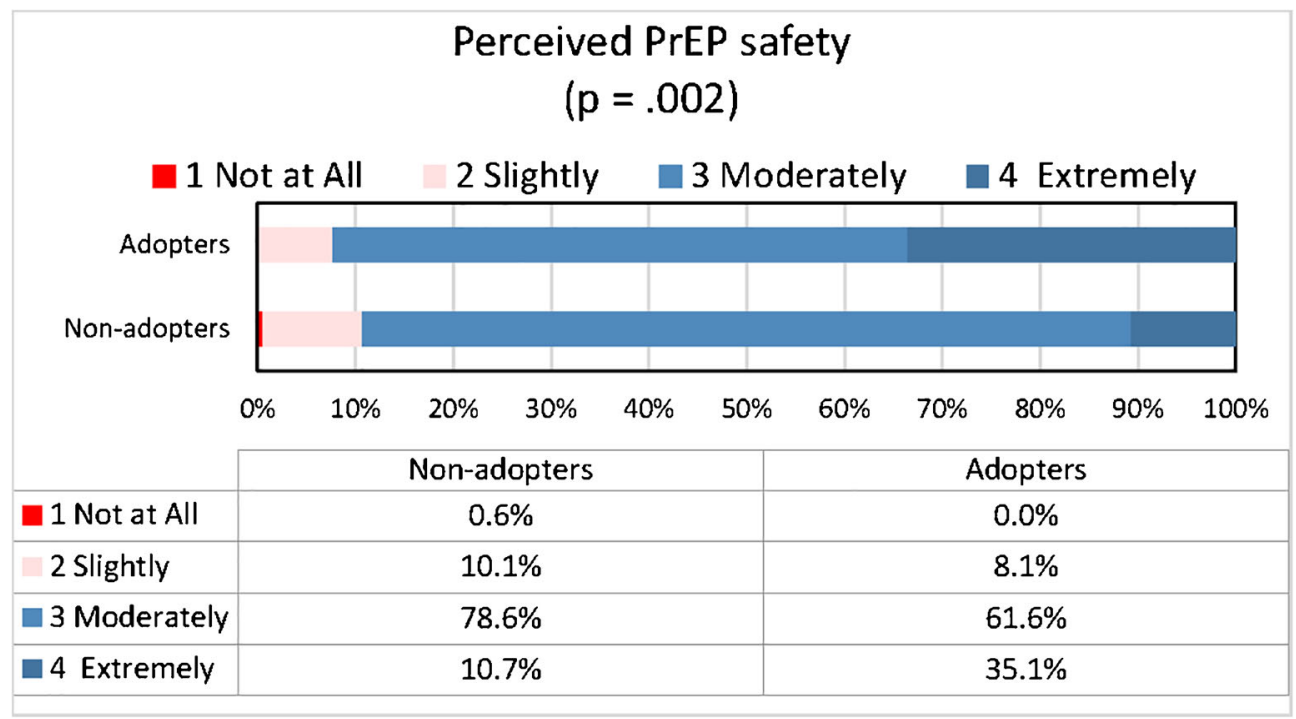

Figure 4 Perceived PrEP safety by adoption status.

among providers in prior studies, ${ }^{11,13,15,23,28,30,31}$ despite the lack of evidence of widespread risk compensation in studies investigating the efficacy ${ }^{36,37}$ and effectiveness ${ }^{38,39}$ of PrEP. In our study, we found that non-adopters were more likely than adopters to perceive PrEP use as leading to risk compensation. Prior studies have found either no association or a negative association between providers' belief in risk compensation and proxies for PrEP adoption (e.g., willingness to prescribe PrEP). ${ }^{23,27,30}$ To the best of our knowledge, ours is the only published study to examine the relationship between a belief in risk compensation and actual PrEP adoption among providers in clinical practice. Regarding safety, a number of studies have also demonstrated provider concerns about potential PrEPrelated toxicities; ${ }^{12,13,15,16,28}$ these concerns have been associated with more negative attitudes toward $\operatorname{PrEP}^{23}$ and less intention to prescribe PrEP. ${ }^{29}$

We believe our study's findings have important implications for the role of PCPs in PrEP implementation. First, the proportion of respondents in our sample who reported PrEP adoption was among the highest estimates of any published study on this topic, highlighting the promising role of PCPs in PrEP implementation. The CDC estimates that about 1.2 million persons in the U.S. are eligible for PrEP. ${ }^{9}$ Although we do not know the exact proportion of PCPs who provide care to persons eligible for PrEP, the fact that the vast majority of persons who are eligible are not receiving PrEP underscores the need to increase the number of PCPs offering PrEP to eligible individuals. ${ }^{10}$ Second, as PCPs providing care to greater than $50 \mathrm{HIV}$-positive patients were more likely to report PrEP adoption than those providing no HIV care, provider-focused educational initiatives to enhance PrEP adoption may need to target those without HIV care experience, as well as training those with HIV care experience to be PrEP "clinical champions". Specifically, clinical champions could provide education, such as assessing eligibility, counseling, prescription, and monitoring, help establish PrEP clinical

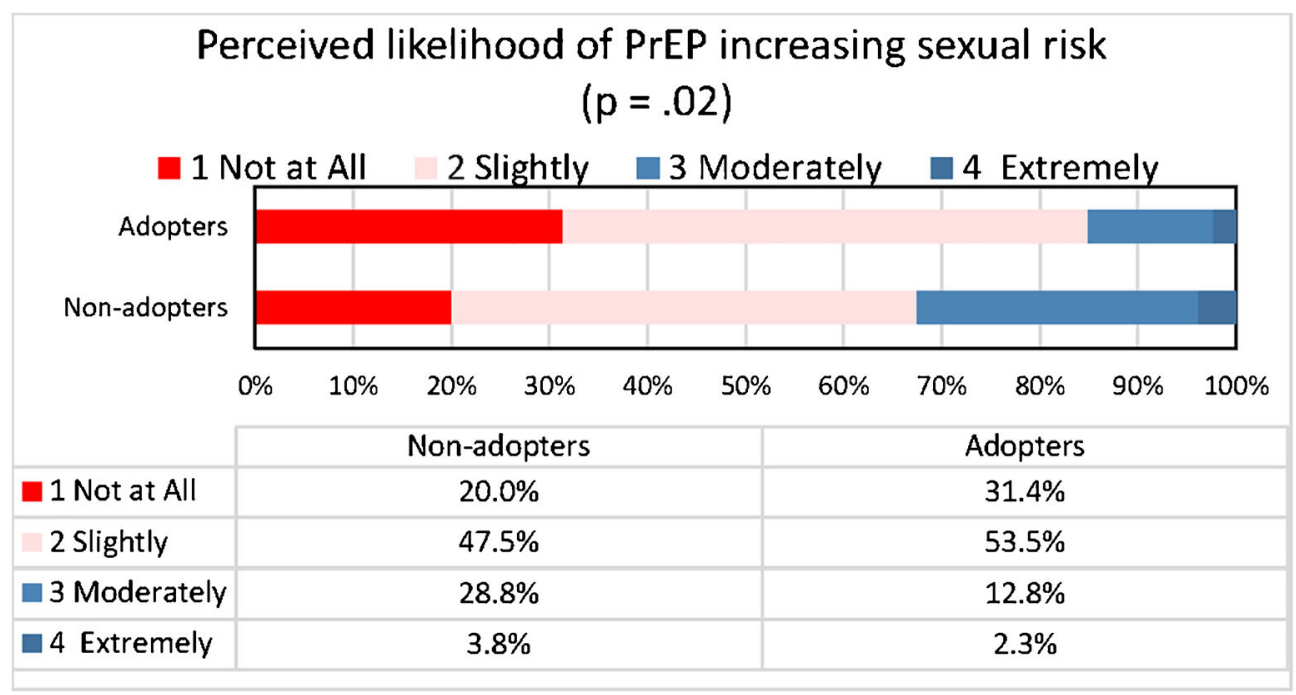

Figure 5 Perceived likelihood of PrEP increasing risk behaviors by adoption status. 


\section{Perceived PrEP effectiveness$$
(p=.20)
$$

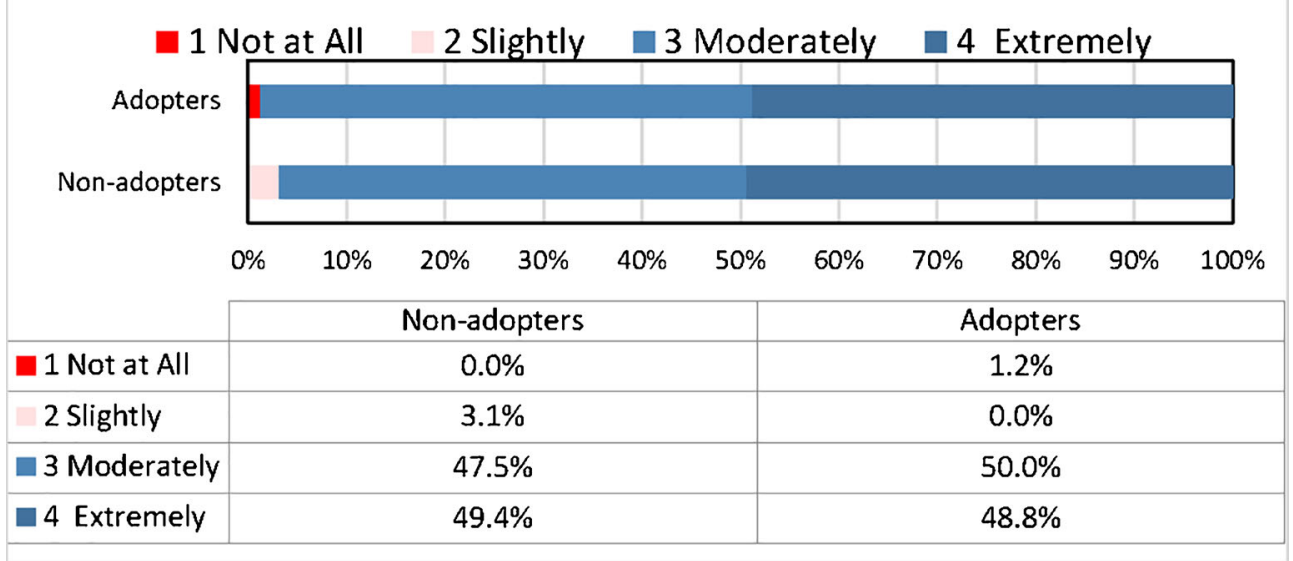

Figure 6 Perceived PrEP effectiveness and safety by adoption status.

practice protocols to facilitate PrEP provision at their local sites, and provide ongoing support to their colleagues as needed. Third, such initiatives will also need to address concerns related to risk compensation and safety. While randomized clinical trials have not observed risk compensation to be normative among participants (measured via self-reported risk behaviors or diagnosed sexually transmitted infections), ${ }^{36-39}$ the perception persists that PrEP will increase risk behaviors, and this was negatively associated with PrEP adoption in our study. Even though increased rates of condomless sex in the context of PrEP use have been reported, this has not been associated with increased risk of HIV acquisition in preliminary accounts of real-world PrEP use. ${ }^{39}$ As PrEP adoption was associated with greater perceived safety, addressing provider concerns about PrEP-related toxicities may also be critically important for adoption efforts.

Despite the strengths of our study, there are limitations that deserve mention. First, we did not specifically ask participants about their patients' risk characteristics. If there were respondents who had not encountered individuals at substantial HIV risk in their clinical practice, then our estimate of adoption may partially reflect a lack of opportunity to prescribe PrEP. However, we believe it is likely that all participants had one or more patients who could have benefited from PrEP based on the applicability of the CDC criteria to diverse patient populations and the estimates that over 1.2 million Americans meet such criteria. Our future research will consider the extent to which PCPs provide care to persons eligible for PrEP relative to PrEP adoption and the potential role for routine screening for PrEP eligibility. Second, our sampling frame may have implications for the generalizability of our findings to academic PCPs and to PCPs more broadly. We recruited a convenience sample of members of a professional society for academic PCPs. Academic PCPs may be more aware of more recent medical advances than those who practice in nonacademic clinical settings. Additionally, although we used both
HIV-specific and general primary prevention messaging for recruitment, PCPs who may be aware of or interested in PrEP, or in HIV prevention and treatment more generally, may have been inclined to participate in the study, thereby affecting the representativeness of our sample. Despite these limitations, which are common among provider attitude surveys of this nature, ${ }^{40,41}$ this study is a foundational step toward characterizing PrEP adoption and related factors among PCPs, who number about 70,000 in the U.S. ${ }^{42}$ Lastly, we obtained a relatively low response rate. However, the rate was similar to that of previously published studies on the topic. ${ }^{13,24}$ Additionally, the exact number of registered SGIM members who met eligibility criteria at the time of the survey is unavailable, as is the number of SGIM members who were active on the listserv (i.e., routinely checking emails). Thus, our calculated response rate is likely a conservative estimate.

In an online survey of PCPs, we found that the vast majority were aware of PrEP, with about one-third of PrEP-aware respondents reporting PrEP adoption. Compared with non-adopters, adopters were more likely to provide care to more than 50 HIV-positive patients and to perceive PrEP as safe. Compared with adopters, non-adopters were more likely to perceive PrEP use as leading to risk compensation. PCP-focused PrEP initiatives should aim to translate these high levels of awareness to PrEP adoption by targeting PCPs without HIV care experience for educational initiatives, as well as training those with experience to be PrEP "clinical champions". Additionally, addressing persistent concerns related to risk compensation and safety may be critical to such efforts. By increasing PrEP adoption, PCPs have substantial potential to contribute to the downward trend in new HIV infections in the U.S.

\section{Acknowledgments:}

Contributors: Theresa Katz, Yale Center for Clinical Investigation, Yale School of Medicine for programming the PCP PrEP survey using survey software. . 
Prior Presentations: Society of General Internal Medicine Annual Meeting, Hollywood, FL, May 11-14, 2016.

Corresponding Author: Oni J. Blackstock, MD, MHS; Montefiore Medical Center/Albert Einstein College of Medicine, 3300 Kossuth Avenue, Bronx, NY 10467, USA (e-mail: oblackst@montefiore.org). Compliance with Ethical Standards:

Funders: This work was generously supported by Yale Center for Clinical Investigation (UL1 TRO00142). E. Jennifer Edelman was funded as a Yale Drug Abuse, Addiction and HIV Research Scholar (DAHRS) during the writing of this manuscript (K12DA033312-03). Oni Blackstock is supported by K23MH102129 and Dr. Sarah Calabrese by K01MH103080.

Conflict of Interest: The authors declare that they do not have a conflict of interest.

\section{REFERENCES}

1. HIV Surveillance Report, Vol 25, Diagnoses of HIV Infection in the United States and Dependent Areas, 2013. (Accessed August 25, 2016, at http:// www.cdc.gov/hiv/pdf/library/reports/surveillance/cdc-hiv-surveillancereport-vol-25.pdf).

2. Preexposure prophylaxis for the prevention of HIV infection in the United States, -2014: A Clinical Practice Guideline. (Accessed August 25, 2016, at http://www.cdc.gov/hiv/pdf/prepguidelines2014.pdf).

3. Baeten JM, Donnell D, Ndase P, et al. Antiretroviral prophylaxis for HIV prevention in heterosexual men and women. N Engl J Med. 2012;367:399-410.

4. Choopanya K, Martin M, Suntharasamai P, et al. Antiretroviral prophylaxis for HIV infection in injecting drug users in Bangkok, Thailand (the Bangkok Tenofovir Study): a randomised, double-blind, placebo-controlled phase 3 trial. Lancet. 2013;381:2083-90.

5. Grant RM, Lama JR, Anderson PL, et al. Preexposure chemoprophylaxis for HIV prevention in men who have sex with men. N Engl J Med. 2010;363:2587-99.

6. Thomson KA, Baeten JM, Mugo NR, Bekker LG, Celum CL, Heffron R. Tenofovir-based oral preexposure prophylaxis prevents HIV infection among women. Curr Opin HIV AIDS. 2016;11:18-26.

7. Fonner VA, Dalglish SL, Kennedy CE, et al. Effectiveness and safety of oral HIV preexposure prophylaxis for all populations. AIDS. 2016;30:197383.

8. Grant R, Mulligan K, McMahan V, Liu AY, Guanira J, Chariyalertsak S, Bekker L, Schechter M, Veloso VG, Glidden DV. Recovery of Bone Mineral Density After Stopping Oral HIV Preexposure Prophylaxis. Boston, MA: Conference on Retroviruses and Opportunistic Infections; 2016.

9. Smith DK, Van Handel M, Wolitski RJ, et al. Vital signs: estimated percentages and numbers of adults with indications for preexposure prophylaxis to prevent HIV acquisition-United States, 2015. MMWR Morb Mortal Wkly Rep. 2015;64:1291-5.

10. Mera R, McCallister S, Palmer B, Mayer G, Magnuson D, Rawlings MK. FTC/TDF (Truvada) for HIV Pre-Exposure Prophylaxis (PrEP) Utilization in the United States: 2012-2015. AIDS 2016 Durban, South Africa; 2016.

11. Castel AD, Feaster DJ, Tang W, et al. Understanding HIV care provider attitudes regarding intentions to prescribe PrEP. J Acquir Immune Defic Syndr. 2015;70:520-8

12. Karris MY, Beekmann SE, Mehta SR, Anderson CM, Polgreen PM. Are we prepped for preexposure prophylaxis (PrEP)? Provider opinions on the real-world use of PrEP in the United States and Canada. Clin Infect Dis. 2014;58:704-12.

13. Krakower DS, Oldenburg CE, Mitty JA, et al. Knowledge, beliefs and practices regarding antiretroviral medications for HIV prevention: results from a survey of healthcare providers in New England. PLoS ONE. 2015; 10:e0132398.

14. Sharma M, Wilton J, Senn H, Fowler S, Tan DH. Preparing for PrEP: perceptions and readiness of Canadian physicians for the implementation of HIV pre-exposure prophylaxis. PLoS ONE. 2014;9:e105283.

15. Tellalian D, Maznavi K, Bredeek UF, Hardy WD. Pre-exposure prophylaxis (PrEP) for HIV infection: results of a survey of HIV healthcare providers evaluating their knowledge, attitudes, and prescribing practices. AIDS Patient Care STDs. 2013;27:553-9.

16. White JM, Mimiaga MJ, Krakower DS, Mayer KH. Evolution of Massachusetts physician attitudes, knowledge, and experience regarding the use of antiretrovirals for HIV prevention. AIDS Patient Care STDs. 2012;26:395-405.

17. Smith DK, Mendoza MC, Stryker JE, Rose CE. PrEP awareness and attitudes in a national survey of primary care clinicians in the United States, 2009-2015. PLoS ONE. 2016;11:e0156592.

18. Damschroder LJ, Aron DC, Keith RE, Kirsh SR, Alexander JA, Lowery JC. Fostering implementation of health services research findings into practice: a consolidated framework for advancing implementation science. Implement Sci. 2009;4:50.

19. Artenie AA, Jutras-Aswad D, Roy E, et al. Visits to primary care physicians among persons who inject drugs at high risk of hepatitis C virus infection: room for improvement. J Viral Hepat. 2015;22:792-9.

20. Brownson RC, Colditz G, Proctor EK. Dissemination and Implementation Research in Health: Translating Science to Practice. New York: Oxford University Press; 2012.

21. Krakower DS, Beekmann SE, Polgreen PM, Mayer KH. Diffusion of newer HIV prevention innovations: variable practices of frontline infectious diseases physicians. Clin Infect Dis. 2016;62:99-105.

22. Krakower D, Ware N, Mitty JA, Maloney K, Mayer KH. HIV providers' perceived barriers and facilitators to implementing pre-exposure prophylaxis in care settings: a qualitative study. AIDS Behav. 2014;18:1712-21.

23. Puro V, Palummieri A, De Carli G, Piselli P, Ippolito G. Attitude towards antiretroviral pre-exposure prophylaxis (PrEP) prescription among HIV specialists. BMC Infect Dis. 2013;13:217.

24. Adams LM, Balderson BH. HIV providers' likelihood to prescribe preexposure prophylaxis (PrEP) for HIV prevention differs by patient type: a short report. AIDS Care. 2016;28(9):1154-8.

25. Hoffman S, Guidry JA, Collier KL, et al. A clinical home for preexposure prophylaxis: diverse health care providers' perspectives on the "purview paradox". J Int Assoc Providers AIDS Care. 2016;15:59-65.

26. Arnold EA, Hazelton P, Lane T, et al. A qualitative study of provider thoughts on implementing pre-exposure prophylaxis (PrEP) in clinical settings to prevent HIV infection. PLoS ONE. 2012;7:e40603.

27. Tripathi A, Ogbuanu C, Monger M, Gibson JJ, Duffus WA. Preexposure prophylaxis for HIV infection: healthcare providers' knowledge, perception, and willingness to adopt future implementation in the southern US. South Med J. 2012;105:199-206.

28. Blumenthal J, Jain S, Krakower D, et al. Knowledge is power! Increased provider knowledge scores regarding pre-exposure prophylaxis (PrEP) are associated with higher rates of PrEP prescription and future intent to prescribe PrEP. AIDS Behav. 2015;19:802-10.

29. Mimiaga MJ, White JM, Krakower DS, Biello KB, Mayer KH. Suboptimal awareness and comprehension of published preexposure prophylaxis efficacy results among physicians in Massachusetts. AIDS Care. 2014;26:684-93.

30. Desai M, Gafos M, Dolling D, McCormack S, Nardone A, PROUD Study. Healthcare providers' knowledge of, attitudes to and practice of preexposure prophylaxis for HIV infection. HIV Med. 2016;17:133-42.

31. Tang EC, Sobieszczyk ME, Shu E, Gonzales P, Sanchez J, Lama JR. Provider attitudes toward oral preexposure prophylaxis for HIV prevention among high-risk men who have sex with men in Lima, Peru. AIDS Res Hum Retrovir. 2014;30:416-24.

32. Seidman D, Carlson K, Weber S, Witt J, Kelly PJ. United States family planning providers' knowledge of and attitudes towards preexposure prophylaxis for HIV prevention: a national survey. Contraception. 2016;93:463-9.

33. Lum PJ, Little S, Botsko $\mathbf{M}$, et al. Opioid-prescribing practices and provider confidence recognizing opioid analgesic abuse in HIV primary care settings. J Acquir Immune Defic Syndr. 2011;56(Suppl 1):S91-7.

34. Mimiaga MJ, Reisner SL, Grasso C, et al. Substance use among HIVinfected patients engaged in primary care in the United States: findings from the Centers for AIDS Research Network of Integrated Clinical Systems cohort. Am J Public Health. 2013;103:1457-67.

35. Edelman EJTJ, Fiellin DA. Substance use in older HIV-infected patients. Curr Opin HIV AIDS. 2014;9:317-24.

36. Marcus JL, Glidden DV, Mayer KH, et al. No evidence of sexual risk compensation in the iPrEx trial of daily oral HIV preexposure prophylaxis. PLOS ONE. 2013;8:e81997.

37. Mugwanya KK, Donnell D, Celum C, et al. Sexual behaviour of heterosexual men and women receiving antiretroviral pre-exposure prophylaxis for HIV prevention: a longitudinal analysis. Lancet Infect Dis. 2013;13:1021-8.

38. McCormack S, Dunn DT, Desai M, et al. Pre-exposure prophylaxis to prevent the acquisition of HIV-1 infection (PROUD): effectiveness results from the pilot phase of a pragmatic open-label randomised trial. Lancet. 2016;387:53-60. 
39. Volk JE, Marcus JL, Phengrasamy T, et al. No new HIV infections with increasing use of HIV preexposure prophylaxis in a clinical practice setting. Clin Infect Dis. 2015;61:1601-3.

40. Dykema J, Stevenson J, Day B, Sellers SL, Bonham VL. Effects of incentives and prenotification on response rates and costs in a national web survey of physicians. Eval Health Prof. 2011;34:434-47.
41. Klabunde CN, Willis GB, McLeod CC, et al. Improving the quality of surveys of physicians and medical groups: a research agenda. Eval Health Prof. 2012;35:477-506.

42. The Number of Practicing Primary Care Physicians in the United States. (Accessed August 25, 2016, at http://www.ahrq.gov/research/findings/ factsheets/primary/pcwork1/index.html). 